\title{
Baryogenesis at Low Reheating Temperatures
}

\author{
Sacha Davidson ${ }^{(1)}$, Marta Losada $^{(2)}$ and Antonio Riotto ${ }^{(3,4)}$ \\ (1) Theoretical Physics, Oxford University, 1 Keble Road, Oxford, OX1 3NP, UK \\ ${ }^{(2)}$ Centro de Investigaciones, Universidad Antonio Nariño, Cll. 57 No. 37-71, Santa Fe de Bogotá, Colombia \\ ${ }^{(3)}$ Scuola Normale Superiore, Piazza dei Cavalieri 7, I-56126 Pisa, Italy \\ (4) INFN, Sezione di Pisa, I-56127 Pisa, Italy
}

(January, 2000)

\begin{abstract}
We note that the maximum temperature during reheating can be much greater than the reheating temperature $T_{r}$ at which the Universe becomes radiation dominated. We show that the Standard Model anomalous $(B+L)$-violating processes can therefore be in thermal equilibrium for $1 \mathrm{GeV}$ $\lesssim T_{r} \ll 100 \mathrm{GeV}$. Electroweak baryogenesis could work and the traditional upper bound on the Higgs mass coming from the requirement of the preservation of the baryon asymmetry may be relaxed. Alternatively, the baryon asymmetry may be reprocessed by sphaleron transitions either from a $(B-L)$ asymmetry generated by the Affleck-Dine mechanism or from a chiral asymmetry between $e_{R}$ and $e_{L}$ in a $B-L=0$ Universe. Our findings are also relevant to the production of the baryon asymmetry in large extra dimension models.
\end{abstract}

PACS: 98.80.Cq; SNS-PH/00-01

Introduction. Theories that explain the tiny difference between the number density of baryons and antibaryons - about $10^{-10}$ if normalized to the entropy density of the Universe - represent perhaps the best example of the interplay between particle physics and cosmology. Until now, many mechanisms for the generation of the baryon asymmetry have been proposed [1]. Baryogenesis at the electroweak scale has been of recent interest, and is attractive because it can be tested at current and future accelerator experiments. On the other hand, we know that the flatness and the horizon problems of the standard big bang cosmology are elegantly solved if during the evolution of the early Universe the energy density is dominated by some form of vacuum energy, and comoving scales grow quasi-exponentially [2]. This naturally generates the observed large scale density and temperature fluctuations. This inflationary stage can be parametrised by the evolution of some scalar field $\phi$, the inflaton, which is initially displaced from the minimum of its potential. Inflation ends when the potential energy associated with the inflaton field becomes smaller than the kinetic energy of the field. The low-entropy cold Universe dominated by the energy of coherent motion of the $\phi$ field must then be transformed into a high-entropy hot Universe dominated by radiation. This process has been dubbed reheating. Of particular interest is a quantity known as the reheating temperature $T_{r}$, defined such that the energy density of the Universe when it becomes dominated by radiation is $\propto T_{r}^{4}$. Notice that the Universe might have gone through further processes of reheating if - after inflation - the energy density of the Universe happened to be dominated by the the coherent oscillations of some generic weaklycoupled scalar fields, e.g. some moduli fields which are ubiquitous in string and supersymmetric theories.

A common assumption in baryogenesis models is that the post-inflationary Universe contained a plasma in thermal equilibrium with initial temperature $T$ much larger than (or at least of order of) the electroweak scale. This is required to have acceptable initial conditions for the most popular baryogenesis mechanisms, and to take advantage of the Standard Model (SM) anomalous $(B+L)$-violation.

This assumption seems so natural that it is rarely questioned. However, low reheating temperature scenarios are particularly welcome if one wishes to avoid the overproduction of dangerous relics at (pre)heating stage [3] after inflation (such as gravitinos and moduli fields), or at reheating (gravitons in models with large extra dimensions (4]). Apart from these speculative arguments, we do not know the history of the observable Universe before the epoch of nucleosynthesis - all we know experimentally is that $T_{r} \gtrsim 1 \mathrm{MeV}$.

The three required ingredients for baryogenesis are baryon number violation, $\mathrm{C}$ and $\mathrm{CP}$ violation and outof-equilibrium dynamics. It is not easy to generate the baryon asymmetry in a Universe that reheats to a low temperature because the first and third ingredients are hard to come by [5]: it is difficult to introduce baryon number violation at low temperatures without contradicting laboratory bounds on $B$ violation, and the Universe is expanding so slowly at low temperatures that it is very close to equilibrium. There are nonetheless some models for baryogenesis in cold Universes [5,6].

The possibility of using anomalous electroweak $\Delta B=$ $\Delta L=3$ operators to generate the baryon asymmetry in a low $T_{r}$ Universe is particularily interesting for Low Quantum Gravity Scale (LQGS) models [4]. In these theories, the $(4+n)$-dimensional string scale $M_{s}$ is well below the $4 D$ Planck mass $M_{p}$. Gravity is weak on our 4-dimensional brane because it is "diluted" in the $n$ compact dimensions where ordinary matter cannot propagate. The usual baryogenesis mechanisms [5] are diffi- 
cult to implement in these theories because the reheat temperature on our brane must be low to avoid overproducing gravitons in the large extra dimensions, and because the laboratory bounds on baryon number violation are significant. If every operator not forbidden by a gauge symmetry is generated at the quantum gravity scale with a coefficient of order unity, then $\Delta B=1$ operators capable of mediating proton decay need to be forbidden for $M_{s} \lesssim\left(10^{9}-10^{26}\right) \mathrm{GeV}$ [5]. Neutron-antineutron oscillations can be generated by $\Delta B=2$ operators, which must be forbidden for $M_{s} \lesssim 10^{5} \mathrm{GeV}$.

The aim of the present Letter is to show that baryogenesis is much less difficult than anticipated in a Universe with a low reheating temperature (say much below the electroweak scale). Contrary to naive expectations, baryogenesis scenarios using electroweak $(B+L)$ violation remain viable. We will show that electroweak $(B+L)$-violating processes may be present even though $T_{r} \ll 100 \mathrm{GeV}$. This is already a surprising result. Furthermore, electroweak baryogenesis is possible and the traditional upper bound on the Higgs mass coming from the requirement of the preservation of the baryon asymmetry is relaxed because the Universe is expanding faster so sphaleron configurations go easily out of equilibrium after the electroweak phase transition (EPT) ${ }^{*}$. Alternatively, the anomalous $(B+L)$-violation may reprocess an asymmetry in $(B-L)$ generated by some other mechanism, for instance Affleck-Dine [8]. We will also show that the electron Yukawa coupling can be out of equilibrium while the sphalerons are present, so a primordial asymmetry between $e_{R}$ and $e_{L}$ in a $B-L=0$ Universe can be transformed by the $(B+L)$-violation into a baryon asymmetry 9 .

Details of the reheating stage. We now discuss the key argument of our idea. All our considerations are based on the fact that reheating is far from being an instantaneous process. This is a simple, but crucial point [12,13.

Suppose reheating is due to the perturbative decay of a weakly-coupled scalar field $\phi$. The latter might be the inflaton field as well as a modulus. The radiationdominated phase follows a prolonged stage of coherent oscillations of $\phi$. During the epoch between the initial time $H_{I}^{-1}$ (the time at which the oscillations start) and the time of reheating $\Gamma_{\phi}^{-1}$, where $\Gamma_{\phi} \equiv \alpha_{\phi} M_{\phi}$ is the decay rate of the field, the energy density per unit comoving volume of the scalar field $\phi$ decreases slowly as $e^{-\Gamma_{\phi} t}$ while $\phi$ decays into lighter states. For low reheat temperatures, the decay products of the scalar field thermalize rapidly [10,11]. As the coherent $\phi$ oscillations gradually decay, the temperature of the Universe does not scale as $T \sim a^{-1}$ (as in the radiation-dominated era), but follows a different law 12,10]: $T=T_{m} f(a)$. Here

\footnotetext{
*see [7] for a general phenomenological discussion of nonstandard cosmologies where the sphaleron bound is weakened.
}

$$
T_{m}=0.54 \frac{g_{*}^{1 / 8}\left(T_{r}\right)}{g_{*}^{1 / 4}\left(T_{m}\right)}\left(M_{p} H_{I}\right)^{1 / 4} T_{r}^{1 / 2} \simeq \frac{T_{r}}{\alpha_{\phi}^{1 / 4}}
$$

and

$f(a) \equiv K\left(a^{-3 / 2}-a^{-4}\right)^{1 / 4}, K \equiv 1.3\left(g_{*}\left(T_{m}\right) / g_{*}(T)\right)^{1 / 4}$. The function $f(a)$ grows until $a_{0}=(8 / 3)^{2 / 5}$, where it reaches its maximum $f\left(a_{0}\right)=1$, and then decreases as $f \sim K a^{-3 / 8}$. Therefore, for $a>a_{0}$, the temperature can be approximated by $T \simeq T_{m} K a^{-3 / 8}$. This result shows that, during the phase before reheating, the temperature reaches a maximum temperature $T_{m}$ and then has a less steep dependence on the scale factor $a$ than in the radiation-dominated era. The Hubble rate is

$$
H \simeq \sqrt{\frac{8 \pi g_{*}(T)}{3}} \frac{T^{2}}{M_{p}} \frac{g_{*}^{1 / 2}(T) T^{2}}{g_{*}^{1 / 2}\left(T_{r}\right) T_{r}^{2}},
$$

and - at a given temperature - the expansion is faster the smaller is the reheat temperature. Therefore $T_{r}$ is not the maximum temperature obtained in the universe during reheating. Note that this should be qualitatively true of any model with a low $T_{r}$, and does not depend on the details of reheating. The maximum temperature can be much larger than $T_{r}$ provided that $H_{I} \gg T_{r}^{2} / M_{p}$; for instance $T_{m} \sim 10^{5} \mathrm{GeV}$ for $H_{I} \sim 1 \mathrm{TeV}$ and $T_{r} \sim 1 \mathrm{GeV}$. This means that anomalous $(B+L)$-violation may be in equilibrium even though the reheat temperature is very low. We also see that for temperatures larger than $T_{r}$, the expansion rate is faster than for a radiation-dominated Universe at a given temperature $T$.

Electroweak baryogenesis. The fundamental idea of electroweak baryogenesis is to produce asymmetries in some local charges which are (approximately) conserved by the interactions inside the walls of the expanding bubbles formed during the EPT. Local departure from thermal equilibrium is attained inside the walls. Local charges diffuse into the unbroken phase where baryon number violation is active thanks to the unsuppressed $(B+L)$-violation [14. This converts the asymmetries into baryon asymmetry, because the state of minimum free energy is attained for nonvanishing baryon number. Finally, the baryon number flows into the broken phase where it would be erased by unsuppressed sphaleron transitions unless $\left\langle h\left(T_{c}\right)\right\rangle / T_{c} \gtrsim 1$, where $\left\langle h\left(T_{c}\right)\right\rangle$ is the vacuum expectation value of the Higgs field at the critical temperature $T_{c} \sim 100 \mathrm{GeV}[15]$. Naively one expects that the bound $\left\langle h\left(T_{c}\right)\right\rangle / T_{c} \gtrsim 1$ - obtained supposing that the electroweak phase transition takes place in a radiationdominated phase - to translate into an upper bound on the Higgs mass in the SM or its extensions. For the SM, two-loop perturbative results give an upper bound in the Higgs mass $m_{h} \lesssim 45 \mathrm{GeV}$. However, nonperturbative results give the drastically different conclusion that no Higgs mass can satisfy the above bound for a top mass $m_{t}=175 \mathrm{GeV}$ [16. In the Minimal Supersymmetric Standard Model (MSSM), given the current LEP bound on the Higgs mass, the so-called light-stop mechanism is 
required to have sphaleron transitions out of equilibrium in the broken phase [17. Thus, the Higgs mass and the lightest stop mass define the allowed region in parameter space. However, we emphasize that recent analysis have shown that the largest allowed Higgs mass is obtained from zero temperature radiative corrections and the upper bound on the Higgs mass from the sphaleron constraint is no longer in effect as long as one has a sufficiently light stop $m_{\tilde{t}} \lesssim 170 \mathrm{GeV}$ [18].

Let us now suppose that the reheating temperature $T_{r} \ll T_{c}$. As we have seen in the previous section, the hot thermal bath may nonetheless reach temperatures $T_{m} \gg T_{c}$. This means that the EPT may well proceed before the Universe has entered the radiation-dominated phase when reheating is completed. The only difference is that the transition takes place in a matter-dominated Universe whose expansion rate is given by Eq. (2). Electroweak baryogenesis may occur even when $T_{r} \ll T_{c}$. This is a nontrivial result. The generation of the baryon asymmetry is mediated by sphaleron transitions in the unbroken phase, at a rate $\Gamma_{s} \simeq k \alpha_{W}^{4} T$, where $k \simeq 0.1(\sim$ few $\times \alpha_{W}$ ] [19]. They are in equilibrium at temperatures $T \lesssim\left(\alpha_{W}^{4} M_{p} T_{r}^{2}\right)^{1 / 3} \sim 10^{4}\left(T_{r} / 1 \mathrm{GeV}\right)^{2 / 3} \mathrm{GeV}$.

Let us now elaborate on the erasure condition. We would like to show that the requirement that sphalerons be out-of-equilibrium in the broken phase is more easily satisfied if $T_{r} \ll T_{c}$ than in the standard cosmology. This is a particular case of the analysis in [7]. At finite temperature $T$ the rate $\Gamma_{s}$ per unit time and unit volume for fluctuations between neighboring minima with different baryon number is 20] $\Gamma_{s} \sim 10^{5} T^{4}\left(\frac{\alpha_{W}}{4 \pi}\right)^{4} \kappa \frac{\zeta^{7}}{B^{7}} e^{-\zeta}$, where $\zeta(T)=E_{s}(T) / T, E_{s}(T)=\left[2 m_{W}(T) / \alpha_{W}\right] B\left(\lambda / g^{2}\right)$ is the sphaleron energy, $m_{W}(T)=\frac{1}{2} g\langle h(T)\rangle, B \simeq 1.9$ is a function which depends weakly on the gauge and the Higgs quartic couplings $g$ and $\lambda, \alpha_{W}=g^{2} / 4 \pi=0.033$. Requiring $\Gamma_{s} / T^{3} \lesssim H$ at the bubble nucleation temperature $T_{b}$ leads to the condition on $\zeta\left(T_{b}\right)$,

$$
\zeta\left(T_{b}\right) \gtrsim 7 \log \zeta\left(T_{b}\right)+9 \log 10+\log \kappa+2 \log \left(T_{r} / T_{b}\right),
$$

where $H$ is given in Eq. (2). This inequality is the standard one [1, 19], with one crucial difference: the presence of the last term. The latter tells us that, if the reheating temperature is much smaller than $T_{c}$ (or equivalently the Universe is expanding very quickly) sphalerons go out-of-equilibrium with ease or they are never in equilibrium in the broken phase! This is one of the main results of our paper. If we assume that $\zeta\left(T_{b}\right) \simeq 1.2 \zeta\left(T_{c}\right)$ [16], then for $\kappa=10^{-1}$ and $T_{r} \sim 1(10) \mathrm{GeV}$, we obtain that $\zeta\left(T_{c}\right) \gtrsim 28(33)$, which translates into

$$
\frac{\left\langle h\left(T_{c}\right)\right\rangle}{T_{c}} \gtrsim 0.77(0.92) .
$$

This bound has to be compared to the standard result $\left\langle h\left(T_{c}\right)\right\rangle / T_{c} \gtrsim 1$ obtained for the same value of $\kappa$. This finding clearly enlarges the available region in parameter space where the sphaleron bound is satisfied and relaxes the upper bound on the stop mass in the MSSM and on the Higgs mass in other extensions of the SM. The implication for the SM is that although current LEP bounds on the Higgs mass still rule out electroweak baryogenesis, for small values of the Higgs mass the phase transition is now strong enough for sphaleron transitions to be suppressed. From the lattice results of Ref. [16] we can determine that Eq. (4) implies that the EPT would be strong enough for baryogenesis for $m_{h} \lesssim 50 \mathrm{GeV}$. More interesting, for the MSSM in the region of allowed Higgs masses the new bound of Eqn (4) could increase the upper bound on the stop mass by about $10 \mathrm{GeV}$ to $m_{\tilde{t}} \lesssim 180 \mathrm{GeV}$ for all other parameters fixed. These and other issues are now under investigation [21].

One should not claim victory too soon, though. While preserving a baryon asymmetry is easier if $T_{r} \ll T_{c}$, the continous decays of the scalar field $\phi$ dump entropy into the thermal soup from $T_{c}$ to $T_{r}$. Indicating by $B_{c}$ the baryon asymmetry to entropy density ratio $n_{B} / s$ generated at the EPT, one finds that the final baryon asymmetry is 13]

$$
\frac{n_{B}}{s} \sim B_{c}\left(\frac{T_{r}}{T_{c}}\right)^{5}
$$

This means that, for $T_{r} \sim 10 \mathrm{GeV}$, the mechanism of baryogenesis at the electroweak scale has to be more efficient by a factor $\sim 10^{5}$ than in the standard case. This is certainly challenging, but not impossible to achieve. Parametrizing $B_{c} \sim \kappa \alpha_{W}^{4} \delta_{C P} f\left(v_{w}\right)$, one would need the CP-violating phases $\delta_{C P}$ and the velocity of the bubble walls $v_{w}$ to be of order of unity [21].

Reprocessing a pre-existing asymmetry. An alternative to electroweak baryogenesis when $T_{r}$ is low is to make use of the anomalous electroweak $(B+L)$-violation to transform a pre-existing asymmetry in $(B-L)_{L}$ into a baryon asymmetry [22]. The Affleck-Dine mechanism [8] is particularly attractive in our framework since it can naturally generate a lepton asymmetry when the slepton fields along the flat directions relax to their minima [23]. This happens when the Universe is still dominated by the $\phi$-oscillations and the hot plasma is still at temperatures much larger than $T_{r}$. The initial lepton asymmetry can naturally be of order unity, it gets reprocessed into baryon asymmetry by sphaleron interactions and is subsequently reduced to the observed value by the large entropy production [21,24.

A further and new possibility is that the sphalerons can reprocess a pre-existing asymmetry between the $e_{L}$ and $e_{R}$ into a baryon asymmetry [9]. This is interesting because the only $B$ or $L$ violation required is the $\mathrm{SM}$ sphalerons, but the out-of equilibrium and CP violation required to generate an asymmetry can take place somewhere other than at the EPT. The idea is that the Universe starts with $B=L=0$, and an excess of $e_{R}$ over anti- $e_{R}$ is created during the $\phi$-oscillations. The Universe is electrically neutral, so there must be asymmetries among other charged particles to compensate the 
$e_{R}$ charge density. The electron Yukawa is small, so the $e_{R}$ remain out of chemical equilibrium until late times. The anomalous SM $(B+L)$-violation is rapid, and acts only on left-handed particles, among which there is a lepton number deficit. This asymmetry in $L_{L}$ will therefore be partially transformed into a baryon asymmetry. If the $(B+L)$-violating processes go out of equilibrium before the $e_{R}$ comes into chemical equilibrium, then this baryon asymmetry will be preserved. In the standard cosmology, this is not the case: the sphalerons go out of equilibrium at or just after the electroweak phase transition, and the electron Yukawa comes into equilibrium before this at temperatures $\sim(10-100) \mathrm{TeV}$ 9,25. However, in our scenario, the expansion rate of the Universe is faster, so it could be possible to reprocess an initial chiral asymmetry between $e_{L}$ and $e_{R}$ into a baryon asymmetry. We need to check that the $e_{R}$ are out of chemical equilibrium while the sphalerons are in equilibrium. As previously discussed, there will be $(B+L)$-violation in equilibrium above the electroweak phase transition if $T_{r} \gtrsim 10 \mathrm{MeV}$. We can estimate the rate associated with the electron Yukawa coupling $h_{e}$ to be $\Gamma_{h_{e}} \simeq 10^{-2} h_{e}^{2} T$, in which case $\Gamma_{h_{e}} \gtrsim H$ at $T \lesssim 30\left(T_{r} / \mathrm{GeV}\right)^{2 / 3} \mathrm{GeV}$. So for $T_{r} \lesssim$ a few $\mathrm{GeV}$, we find that the $e_{R}$ do not come into equilibrium until after the sphalerons are out of equilibrium. This estimate suggests that an initial chiral asymmetry between $e_{R}$ and $e_{L}$ in a $B=L=0$ Universe can be reprocessed into a baryon asymmetry. However, the $e_{R}$ may also be brought into chemical equilibrium by anomalous processes, which we will discuss in a subsequent publication [21]. Note that for this mechanism, the only $B$ or $L$ violation required is that already present in the Standard Model, but large amounts of $\mathrm{CP}$ violation or departure from equilibrium are not required at the EPT.

In conclusion, we have shown that the simple observation that - in a Universe with a low reheat temperature $T_{r}$ - the maximum temperature of the thermal bath can be much larger than $T_{r}$ has rich implications for baryogenesis. This is extremely encouraging because, after all, observationally we only know that $T_{r}$ has to be larger than a few $\mathrm{MeV}$ to allow primordial nucleosynthesis.

\section{ACKNOWLEDGMENTS}

We would like to thank Ian Kogan, Misha Shaposhnikov and Carlos Wagner for useful conversations.

[1] For recent reviews, see W. Buchmuller, S. Fredenhagen, hep-ph/0001098; A. Riotto, hep-ph/9807454; A. Riotto and M. Trodden, Annual Reviews of Nuclear and Particle Science 49, 35 (1999); V.A. Rubakov, M.E. Shaposhnikov, Usp. Fiz. Nauk 166493 (1996).
[2] see D. H. Lyth and A. Riotto, Phys. Rept. 314, 1 (1999).

[3] Kallosh, L. Kofman, A. Linde and A. Van Proeyen, hepth/9907124 G. F. Giudice, I. Tkachev and A. Riotto, JHEP 9908, 009 (1999); G. F. Giudice, A. Riotto and I. Tkachev, JHEP 9911, 036 (1999); G. Felder, L. Kofman and A. Linde, hep-ph/9909508.

[4] N. Arkani-Hamed, S. Dimopoulos and G. Dvali, Phys.Rev. D59 086004,1999,

[5] K. Benakli, S. Davidson, Phys. Rev. D60, 025004 (1999)

[6] S. Dimopoulos, L.Hall, Phys. Lett. B196, 135 (1987); A. Pilaftsis, Phys.Rev. D60 , 105023 (1999); G. Dvali, Phys. Lett. B460, 47 (1999); J. Garcia-Bellido, D. Grigorev, A. Kusenko, M. Shaposhnikov, Phys. Rev. D60, 123504 (1999); G. Dvali and G. Gabadadze, Phys. Lett. B460, 47 (1999); L. Krauss and M. Trodden, Phys . Rev. Lett. 83, 1502 (1999).

[7] M. Joyce, T. Prokopec, Phys.Rev. D57, 6022 (1998).

[8] I. Affleck, M. Dine, Nucl.Phys. B249 361, (1985).

[9] B. A. Campbell, S. Davidson, J. Ellis and K. A. Olive, Phys. Lett. B297, 118 (1992)

[10] D.J.H. Chung, E.W. Kolb and A. Riotto, Phys. Rev. D60 063504 (1999), hep-ph/9809453.

[11] K. Enqvist, K. Eskola, Mod. Phys. Lett. A5 1919 (1990).

[12] R. J. Scherrer and M. S. Turner, Phys. Rev. D31, 681 (1985); E. W. Kolb and M. S. Turner, The Early Universe, (Addison-Wesley, Menlo Park, Ca., 1990); G. Lazarides, C. Panagiotakopoulos, Q. Shafi, Phys. Lett. B 197355 (1987); O. Pantano and A. Riotto, Phys. Lett. B307, 49 (1993).

[13] J. McDonald, Phys.Rev. D43 1063 (1991).

[14] V.A. Kuzmin, V.A. Rubakov and M.E. Shaposhnikov, Phys. Lett. B155, 36 (1985).

[15] A.I. Bochkarev, S.V. Kuzmin and M.E. Shaposhnikov, Phys. Rev. D43, 369 (1991).

[16] K. Kajantie, M. Laine, K. Rummukainen and M. Shaposhnikov, Nucl. Phys. B466, 189 (1996).

[17] M. Carena, M. Quiros and C.E.M. Wagner, Phys. Lett. B380 81 (1996); D. Delepine, J.M. Gerard, R. Gonzalez Felipe and J. Weyers, Phys. Lett. B386, 183 (1996); J.R. Espinosa, Nucl. Phys. B475, 273 (1996); B. de Carlos and J.R. Espinosa, Nucl. Phys. B503, 24 (1997); M. Laine and K. Rummukainen, Nucl. Phys. B535, 423 (1998).

[18] M. Carena, M. Quiros and C.E.M. Wagner, Nucl. Phys. B524, 3 (1998); M. Losada, Nucl. Phys. B537, 3 (1999); M. Losada, hep-ph/9905441.

[19] P. Arnold, D. Son and L.J. Yaffe, Phys. Rev. D55, 6264 (1997); P. Arnold, Phys. Rev. D55, 7781 (1997).

[20] L. Carson, Xu Li, L. McLerran and R.-T. Wang, Phys. Rev. D42, 2127 (1990).

[21] S. Davidson, M. Losada and A. Riotto, to appear.

[22] M. Fukugita, T. Yanagida, Phys.Lett. B174, 45 (1986).

[23] B. A. Campbell, S. Davidson, K. A. Olive, Nucl.Phys. B399, 111 (1993).

[24] B. Campbell, M. K. Gaillard, H. Murayama and K. A. Olive, Nucl. Phys. B538, 351 (1999).

[25] J. Cline, K. Kainulainen, K. Olive, Phys. Rev. D49, 6394 (1994). 